\title{
A fast algorithm to compute cohomology group generators of orientable 2-manifolds.
}

\author{
Paweł Dłotko ${ }^{1 *}$ \\ 1 Jagiellonian University, Institute of Computer Science, Kraków, Poland. \\ pawel.dlotko@uj.edu.pl
}

\begin{abstract}
In this paper a fast algorithm to compute cohomology group generators of cellular decomposition of any orientable closed 2-manifold is presented. The presented algorithm is a dual version of algorithm to compute homology generators presented by David Eppstein [12] and developed by Jeff Erickson and Kim Whittlesey [13].
\end{abstract}

Keyworks computational cohomology, cohomology generators, combinatorial 2-manifold.

\section{Introduction}

In a past twenty years computational homology theory has gained a considerable attention in the computer science community. The Betti numbers and homology generators as a rigorously computable tools has been used in many areas of mathematics like dynamical systems [8] and outside for instance in the material sciences [18]. A number of programming libraries [1],[2],[3] and algorithms has been developed for Betti numbers and homology group generators computations. Among others, a big progress has been done on computing the minimal homology and homotopy generators of cellular decomposition of orientable closed 2-manifolds, see [12], [13].

Until recently the computational aspect of cohomology theory has been left apparat. In our opinion the reason was the conceptual difficulty of the cohomology theory. The first algorithm to compute cohomology group generators together with the cup product, which provides a ring structure of cohomology has been presented in [11]. This algorithm is based on a concept of chain contraction. Also an algorithm to compute cohomology generators of $2 \mathrm{~d}$ images by using irregular graph pyramids has been discussed in [20].

Recently the first cohomology group generators of 3 dimensional manifolds with boundary has been shown to be useful in Discrete Geometrical Approach to Maxwell's equations [19]. Also cohomology generators are useful in image context like global mesh parametrization, texture mapping, shape matching and shape morphing [7],[10],[14],[15] just to name some of areas.

The algorithm to compute cohomology generators presented in [19] is based on a number of reduction techniques [16],[17] called shavings, algebraic reduction [5] and standard Smith Normal From computations. Consequently it can be applied to any finite simplicial, cubical or general CW complex. Generality however has its computational cost. Therefore one may wander if in case of 3-dimensional computer graphics where 2-dimensional orientable manifolds are considered there exist quicker way of obtaining cohomology generators. In this paper we are going to present a dual version of Eppstein [12] algorithm which provide cohomology group generators for combinatorial surfaces (i.e. cell decompositions of closed orientable 2-manifolds). Also a modification inspired by [13] to obtain minimal length cohomology generators intersecting in a chosen 2-face is discussed.

In this paper the main focus is given to computing first cohomology group generators of cellular decomposition of 2-manifolds. The reason is because for 2-manifolds zeroth and second cohomology

*The author is supported by MNiSW grant N N206 625439. 
group are known a priori. Later in this paper, without loose of generality, we will work on connected manifolds. In that case the rank of both zeroth and second cohomology group is one. The zeroth cohomology group generator is a map sending every vertex of the manifold to 1 . The second cohomology group generator is a map sending single 2-cell to 1 and all remaining 2-cells to 0 .

The paper is structured as follows: In the Section 2 the preliminaries are provided. In Section 3 the Eppstein algorithm [12] is reviewed. In Section 4 an algorithm to compute cohomology generators is presented. In Section 5 necessary modifications to obtain optimal cohomology generators are discussed. In Section 6 a short complexity analysis of the presented algorithms is given. Finally in Section 7 the conclusions are drawn.

\section{Preliminaries.}

For a sake of brevity we will skip here the classical introduction to homology and cohomology theory. Further on we will follow the standard notation of (co)chains, (co)homology, boundary $\partial$ and coboundary $\delta$ operators. For a standard introduction to (co)homology theory consult [6].

A closed 2-manifold is a topological space in which every point has a neighborhood homeomorphic to $\mathbb{R}^{2}$. As in Colin de Verdière and Lazarus [9] we will work on a combinatorial surfaces $M=(\mathcal{M}, G)$, where $\mathcal{M}$ is a 2 -manifold and $G$ is a weighted graph embedded on $\mathcal{M}$ such that every face of an embedding is a topological disk. The weight of a edge in $G$ is equal to the length of a corresponding path in $\mathcal{M}$.

For a combinatorial surface $M=(\mathcal{M}, G)$ the dual graph $G^{*}=(V, E)$, where $V$ is the set of barycenters of faces of $G$ and a dual edge $e^{*}$ is placed between any two dual vertices the corresponding primal faces of which share an primal edge in $G$.

It is clear, that classical concepts of simplicial or cubical complexes on an 2-manifold are in fact combinatorial surfaces with a graph $\mathrm{G}$ being their 1-skeleton.

In this paper a classical version of Poincaré duality for manifolds is used. The modern approach to this famous theorem based on a notion of cup product [6] is very powerful and simplify proofs a lot. However the cost is the intuitive content of the theorem. This presentation follow the line in [4]. It is standard, that for a 2 -manifold $\mathcal{M}$ meshed by $\bar{M}$ we have $H_{2}(\bar{M}, \mathbb{Z}) \approx \mathbb{Z}$ if $\mathcal{M}$ is orientable and 0 othervise $^{1}$. Let us define a dual cell structure $\overline{M^{*}}$ on 2 -manifold $\mathcal{M}$ with a cell structure $\bar{M}$. The dual cell structure is defined based on a dualization operator $D: \bar{M} \rightarrow \overline{M^{*}}$ As in case of dual graph the barycenters of faces $f \in \bar{M}$ corresponds to the vertices $D(f)$ of $\overline{M^{*}}$. For faces $f_{1}, f_{2} \in \bar{M}$ intersecting in an edge $e=f_{1} \cap f_{2} \in \bar{M}$ two segments of line joining $D\left(f_{1}\right)$ with a barycenter of $e$ and $D\left(f_{2}\right)$ with barycenter of $e$ form together a dual edge $D(e)$. For every vertex $v \in \bar{M}$ let $e_{1}, \ldots, e_{n} \in \bar{M}$ be the edges having $e_{i} \cap v \neq \emptyset$ for every $i \in\{1, \ldots, n\}$. The polygon bounded by $D\left(e_{1}\right), \ldots, D\left(e_{n}\right)$ is by definition $D(v)$ (see Figure 2 where $D(w)$ is depicted). Further let us assume, that each cell structure $\bar{M}$ and $\overline{M^{*}}$ is provided with a fixed orientation of every cell. It is also straightforward, that 1-skeleton of dual cell structure and the dual graph are isomorphic (although not equal). Therefore further those concepts will be used interchangeably.

Note the bijective correspondence between $\bar{M}$ and $\overline{M^{*}}$ provided by the map $D$. Let us take any element $a \in \bar{M}$ such that $\partial a=\sum_{b \in \bar{M}} \alpha_{a b} b$. In this case it is easy to see, that $\langle\delta D(a), D(b)\rangle=\alpha_{a b}$ (The scalar product $\langle a, b\rangle$ is a linear map defined on generators by $\langle a, b\rangle=0$ if $a \neq b$ and $\langle a, a\rangle=1$ ). Therefore the map $\partial: C_{*}(\bar{M}) \rightarrow C_{*}(\bar{M})$ is mapped through $D$ to $\delta: C^{*}\left(\overline{M^{*}}\right) \rightarrow C^{*}\left(\overline{M^{*}}\right)$. This is a general phenomena and it is easy to see, that we can assign to each p-chain $c \in C_{p}(\bar{M})$ a $(n-p)$ chain $D(c) \in C_{n-p}\left(\overline{M^{*}}\right)$ (where $n$ is a dimension of manifold) such that $\delta D(c)=D(\partial c)$ and vice versa. Therefore it is clear, that $D$ induces an isomorphism

$$
H_{p}(\bar{M}) \approx H^{n-p}\left(\overline{M^{*}}\right)
$$

classically called a Poincaré duality.

\footnotetext{
${ }^{1}$ For coefficients in $\mathbb{Z}_{2}$ we always have $H_{2}\left(\bar{M}, \mathbb{Z}_{2}\right) \approx \mathbb{Z}_{2}$. Therefore any 2-manifold is orientable when the coefficients in $\mathbb{Z}_{2}$ are considered. The results presented in this paper are therefore valid for any manifold $\mathcal{M}$ and cohomology computed over $\mathbb{Z}_{2}$.
} 


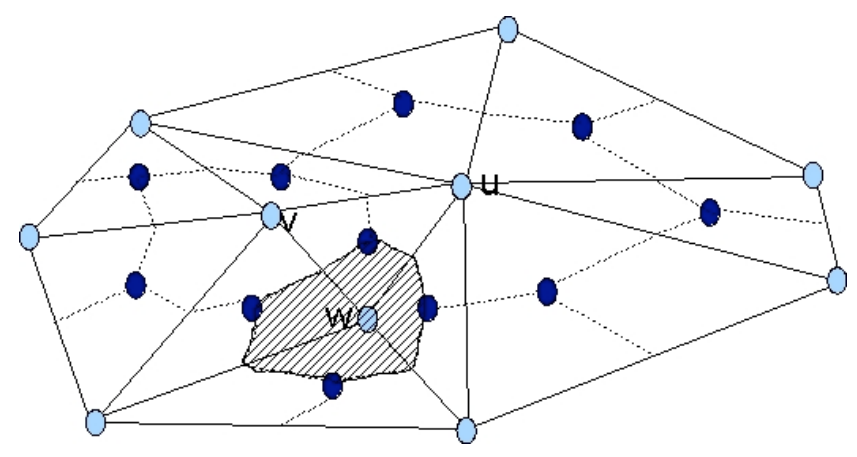

Figure 1: Dual cell structure on a piece of triangulation of 2-manifold. Black dots denote the dual vertices, broken lines - dual edges. With a hatched 2-cell a cell dual to the primal vertex $w$ is presented.

\section{Eppstein algorithm}

In this section the Eppstein algorithm [12] is recalled. For the theoretical background and motivation consult [12]. In this section a pure algorithm and its illustration is provided.

Let $\bar{M}$ be a cell structure on a orientable 2-manifold $\mathcal{M}$ and $\overline{M^{*}}=D(\bar{M})$. The steps of Eppstein algorithm are as follows:

1. Let $T \subset \bar{M}$ be a spanning tree of 1 -skeleton of $\bar{M}$.

2. Let $T^{*} \subset\left(\overline{M^{*}} \backslash \sum_{e \in T} D(e)\right)$ be a spanning tree of 1-skeleton of $\left(\overline{M^{*}} \backslash \sum_{e \in T} D(e)\right)$.

3. Let $E:=\left\{e \in \bar{M}\right.$ such that $e$ is an edge and $e \notin T$ and $\left.D(e) \notin T^{*}\right\}$.

4. For a edge $e \in E$ let $C(e)$ denote the unique cycle in $T \cup e$. The set of cycles $\{C(e) \mid e \in E\}$ forms a basis of the first homotopy group of $M$ (see [13]).

To obtain homology generators one need to construct set of chains the support of which are the cycles $\{C(e)\}_{e \in E}$. This can be easily done by "walking around" a cycle $C(e)$ and assigning to an edge $e$ a value 1 if it is crossed according to its fixed orientation and -1 if it is crossed in the direction opposite to its fixed orientation. The straightforward procedure is illustrated at the Figure 3 .

\section{Algorithm to compute cohomology generators.}

In this section we show that, surprisingly, from Eppstein algorithm one is able to obtain also the cohomology generators.

First let us indicate, that the procedure presented in Section 3 can be run also when the primal and dual cell structures are exchanged provided the map $D^{-1}$ is used instead of the map $D$. In this case, the Eppstein algorithm provide first homology group generators $\left\{c_{i}\right\}_{i=1}^{n}$ in the dual complex $\overline{M^{*}}$.

Let us go back to the Poincaré duality presented in Preliminaries. It implies, that $D^{-1}$ : $C_{*}\left(\overline{M^{*}}\right) \rightarrow C_{*}(\bar{M})$ induces an isomorphism in a Poincaré duality $D_{*}^{-1}: H_{p}\left(\overline{M^{*}}\right) \rightarrow H^{n-p}(\bar{M})$. In our case $n=2$. Let us use this fact for $p=1$. We get, that $D_{*}^{-1}: H_{1}\left(\overline{M^{*}}\right) \rightarrow H^{1}(\bar{M})$ is an isomorphism induced by a map $D^{-1}$. Therefore $\left\{D^{-1}\left(c_{1}\right), \ldots, D^{-1}\left(c_{n}\right)\right\}$ are the cohomology generators of $H^{1}(\bar{M})$.

Moreover, since in the Eppstein algorithm all spanning tree $T$ of $\bar{M}$ and dual spanning tree $T^{*}$ of $\left(\overline{M^{*}} \backslash \bigcup_{t \in T} D(t)\right)$ are acceptable it is easy to see, that the points (1), (2) and (3) of the algorithm presented in Section 3 can be left without any change. Only the point (4) of the algorithm should be replaced by the following set of instructions 

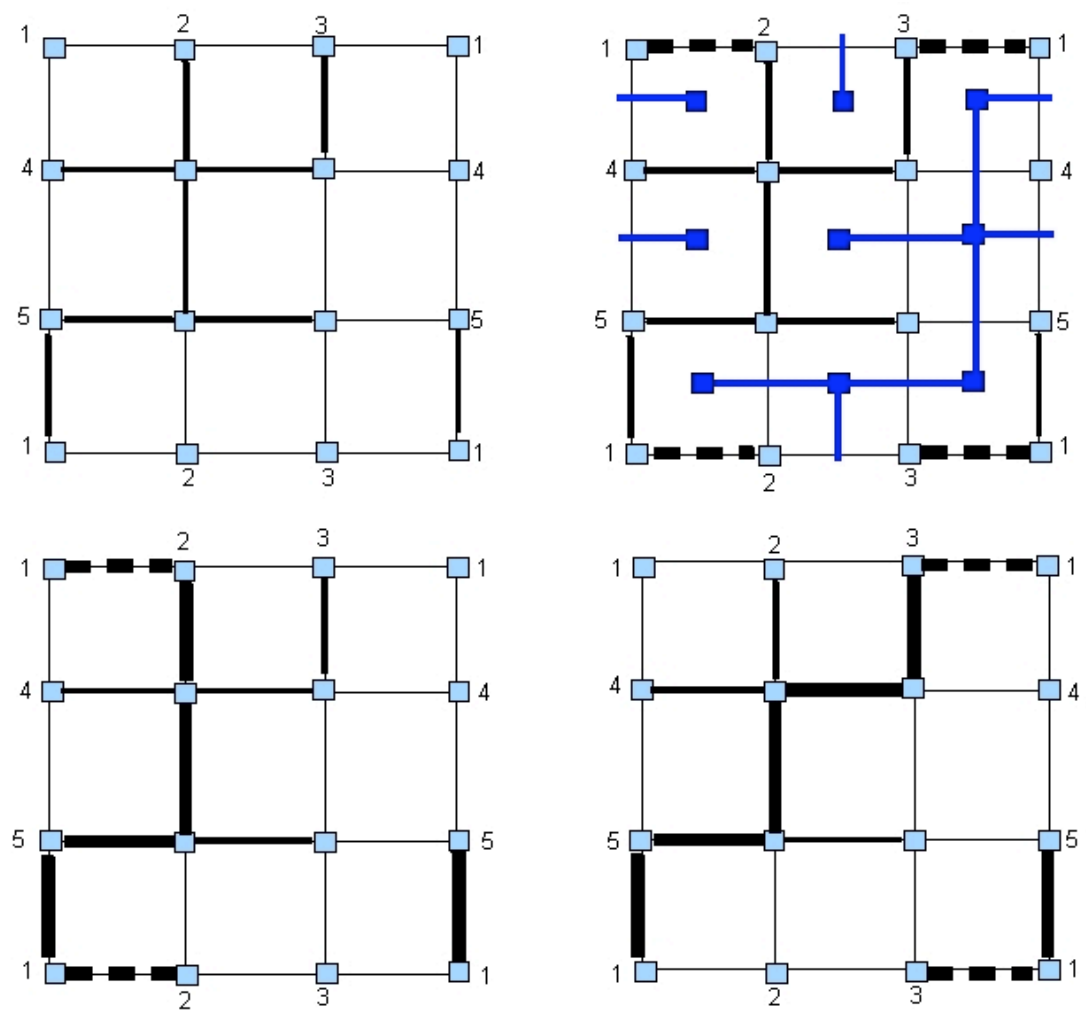

Figure 2: The Eppstein algorithm. On the upper left the spanning tree on a primal complex of a standard cubical representation of a torus. On the upper right the spanning tree on a dual graph is added (in dotted line the edges which are neither in primal nor dual graph). On the bottom row two generators closed by the dotted edges and the tree of the primal complex are indicated with the double bold continuous and dotted edges.

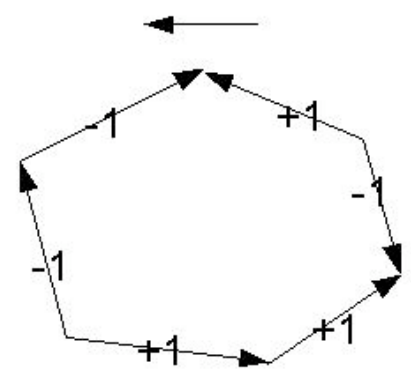

Figure 3: Orientation of a cycle. The arrow at the top points the chosen orientation of the cycle. The numbers about the edges indicate the coefficients of the edge in a constructed chain.

4 For an edge $e \in E$ let $C(D(e))$ denote the unique cycle in $T^{*} \cup D(e)$.

5 For the set of cycles $\{C(D(e)) \mid e \in E\}$ let $h_{1}, \ldots, h_{n} \in Z_{1}\left(\overline{M^{*}}\right)$ be the cycles supported in $C(D(e))$ with coefficients obtained from orientation as in Figure 3. Then $\left\{h_{1}, \ldots, h_{n}\right\}$ represents a basis of $H_{1}\left(\overline{M^{*}}\right)$ (see Section 3$)$. 
6 As the direct consequence of the Poincaré duality the set of cocycles $D^{-1}\left(h_{1}\right), \ldots, D^{-1}\left(h_{n}\right) \in$ $Z^{1}(\bar{M})$ forms basis of $H^{1}(\bar{M})$.

In the Figure 4 the support of the cocycles are indicated.
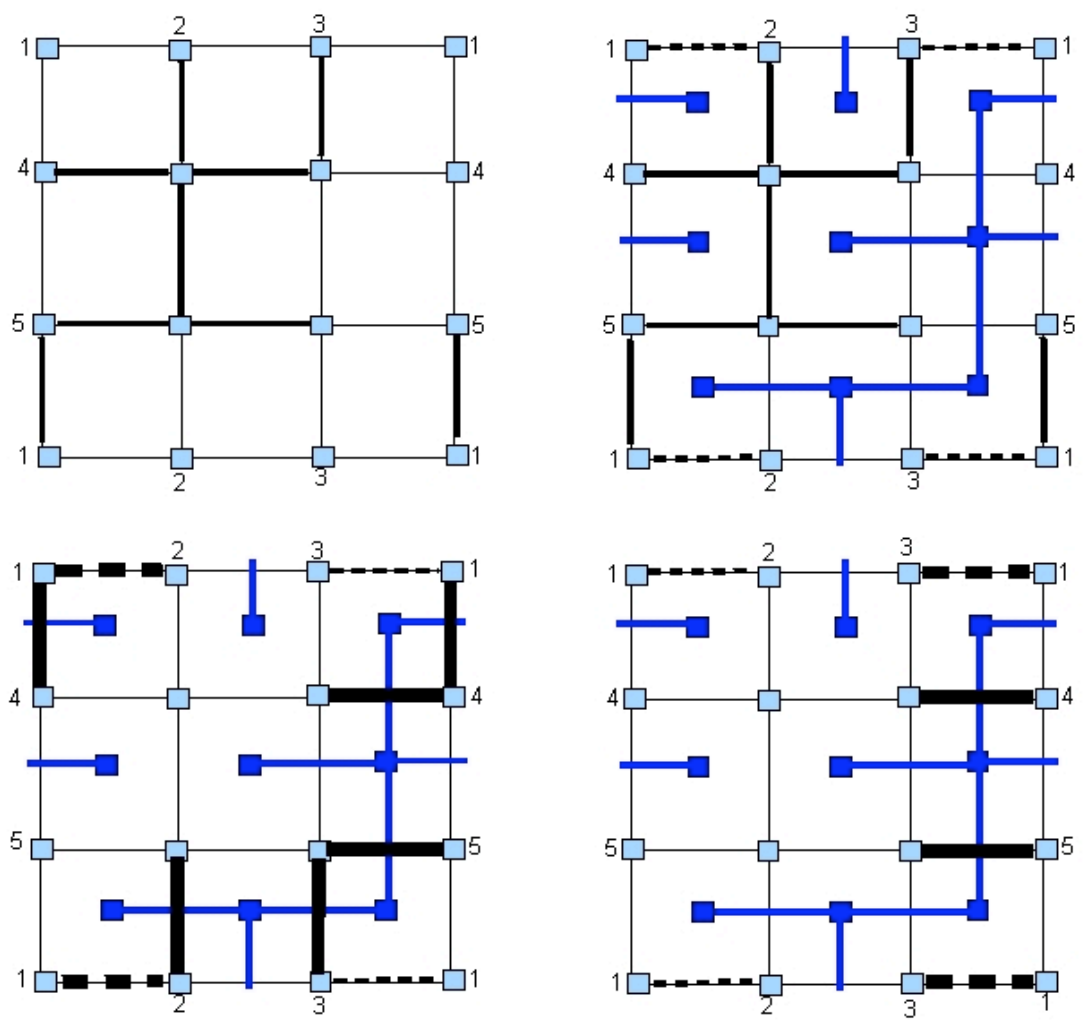

Figure 4: In the upper row - primal and dual tree structure. On the lower row - support of cocycles representing the first cohomology group generators of a torus.

\section{In search of minimality, Erickson and Whittlesey modifi- cation.}

In this section an analogue of Erickson and Whittlesey modification [13] to Eppstein algorithm is discussed. Let us focus here only on minimal generators that intersect on a fixed base point. First let us present a algorithm to obtain minimal length homotopy generators of orientable 2-manifold taken from [13]:

1. Let $T$ be a tree of shortest paths from a chosen vertex $v$ (obtained from Dikstra algorithm) of $\bar{M}$.

2. For each edge $e \in \bar{M} \backslash T$ let $\sigma(e)$ be the length of the unique cycle in $T \cup e$ containing vertex $v$.

3. Let $T^{*}$ be a the maximal spanning tree of $\overline{M^{*}} \backslash \bigcup_{t \in T} D(t)$ where the weight of an edge $D(e) \in \overline{M^{*}} \backslash \bigcup_{t \in T} D(t)$ is $\sigma(e)$.

4. Let $E:=\left\{e \in \bar{M} \mid e \notin T\right.$ and $\left.D(e) \notin T^{*}\right\}$. 
5. For a edge $e \in \bar{M}$ let $C(e)$ denote the unique cycle in $T \cup e$. The set of cycles $\{C(e) \mid e \in E\}$ forms shortest basis of the first homotopy group of $\mathcal{M}$ with the base point $v$ (see [13]).

Similarly as in case of Eppstein algorithm to obtain cohomology group generators it suffice to exchange primal and dual complexes (and use map $D^{-1}$ instead of $D$ ) in the algorithm presented above (with the weight of dual edges equal to the length of the corresponding edges in dual graph). The obvious details of obtaining cocycles from its supports are skipped here. To compleat the section let us present a illustration of this procedure for a standard representation of 2-torus.
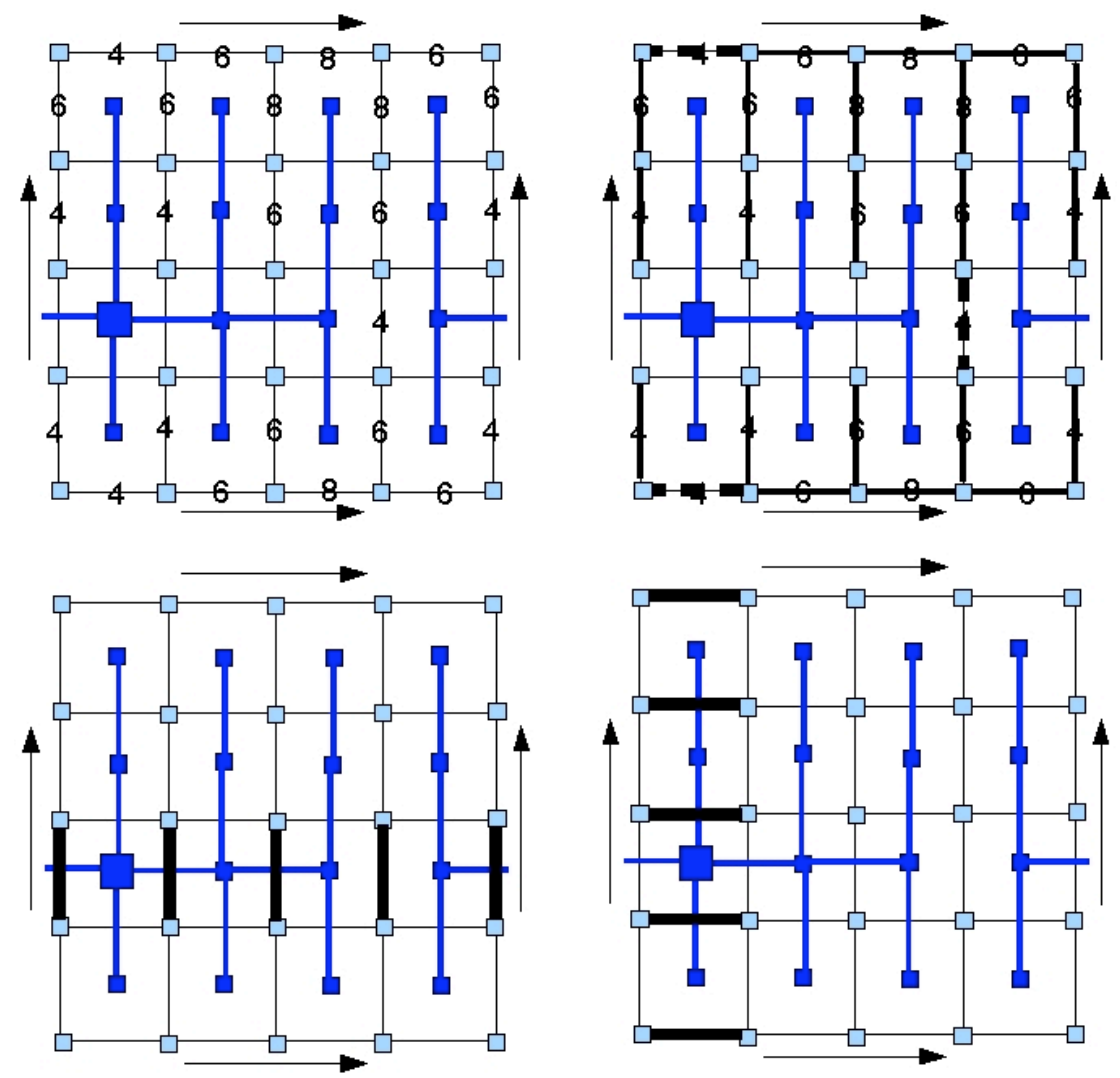

Figure 5: Minimal cohomology basis. In this picture we do not use the convention of naming the vertices in order not to cause a confusion. The general assumption of this example is that all the edges has length 1 . On a top left the shortest path dual tree rooted at the marked 2-cell. The numbers next to the primal edges are their weights. On the top right the maximal spanning tree of the $\overline{M^{*}} \backslash \bigcup_{t \in T} D(t)$. By the dotted edges the edges closing cycles are depicted. On the bottom row the supports of two minimal length cohomology generators are depicted with bold edges.

\section{Complexity.}

In this section the complexity aspects of the algorithms are discussed. First let us indicate, that the concept of dual graph and dual complex used in this paper do not have to be implemented. Therefore to let those algorithms go it suffices to have for instance a pointer representation of a primal complex where each element keeps pointers to its boundary and coboundary elements and extra integer field to store additional information. 
In the case of algorithm presented in Section 4 the spanning trees and the edges in the set $E$ can be found in $O(\operatorname{card}(\bar{M}))$ time. Then the set of cycles can be oriented in a time $O(\operatorname{card}(E) \operatorname{card}(\bar{M}))$. Consequently the whole algorithm has $O(\operatorname{card}(\bar{M})+\operatorname{card}(E) \operatorname{card}(\bar{M}))$ complexity.

In the case of algorithm presented in Section 5 the shortest paths tree can be found by Dikstra'a algorithm in $O(\operatorname{card}(\bar{M}) \log (\operatorname{card}(\bar{M})))$ time. With the same time the dual maximal spanning tree can be found by using for example Kruskal's algorithm. As in the previous case the set of dual cycles can be found in $O(\operatorname{card}(E) \operatorname{card}(\bar{M}))$ time. Consequently the algorithm works in $O(\operatorname{card}(\bar{M}) \log (\operatorname{card}(\bar{M}))+\operatorname{card}(E) \operatorname{card}(\bar{M}))$ time.

\section{Conclusions and further work.}

In this paper a linear algorithm to compute cohomology group basis of a orientable 2-manifold has been provided. In order to obtain the set of shortest generators intersecting in a fixed dual vertex a modification of the algorithm working in $O(\operatorname{card}(\bar{M}) \log (\operatorname{card}(\bar{M}))+\operatorname{card}(E) \operatorname{card}(\bar{M}))$ may be used. Time complexity and general simplicity of the algorithm can make it very useful for the computer graphic community. We would like to point out that, with some additional work ${ }^{2}$, it is also possible to obtain the globally minimal set of cohomology generators over $\mathbb{Z}_{2}$ as it is described in [13].

The technique presented in this paper seems do not have an analogue for higher dimensional manifolds. The reason is, that the 1-skeleton of primal and dual complex gives a complete description of 2-manifold, but are not enough for higher dimensional manifolds.

It seems, that the presented method can be easily extended to 2-manifolds with boundary. In a full version of the paper the extension based on the idea described in [21] will be presented.

\section{References}

[1] The CAPD Library, capd.ii.uj.edu.pl.

[2] The Chomp Library, chomp.reutgers.edu.

[3] The LinBox Library, linalg.org.

[4] G. E. Bredon, Topology and Geometry, Springer-Verlag 1993.

[5] T. Kaczynski, M. Mrozek, M. S̀lusarek, Homology Computation by Reduction of Chain Complexes, Computers and Mathematics 1998.

[6] A. Hatcher, Algebraic topology, Cambridge University Press 2002.

[7] Gu X., Yau S., Computing conformal structures of surfaces, Communications in Information and Systems 2002.

[8] K. Mischaikow, M. Mrozek, Handbook of Dynamical Systems III: Towards Applications, 2002.

[9] E. Colin de Verdiere, F. Lazarus, Optimal pants decompositions and shortest homotopic cycles on an orientable surface, Lecture Notes Comput. Sci. 2003.

[10] Gu X., Wang, Y., Yau S.-T., Multiresolution computation of conformal structures of surfaces, Journal of Systemics, Cybernetics and Informatics 2003.

[11] R. González-Diáz, P. Real, Computation of cohomology operations on finite simplicial complexes, Homology, Homotopy \& Appl. 2003.

\footnotetext{
${ }^{2}$ This additional work is computing the set of generators for every base point and, by solving linear systems of equation, finding set of globally minimal length generators.
} 
[12] D. Eppstein, Dynamic generators of topologically embedded graphs, Proc. 15th ACM-SIAM Sympos. Discrete Algorithms. 2004.

[13] Jeff Erickson, Kim Whittlesey, Greedy optimal homotopy and homology generators, Proc. 16th Ann. ACM-SIAM Symp. Discrete Algorithms 2005.

[14] Guo X., Li X., Bao Y., Gu X., Qin H., Meshless thin-shell simulation based on global conformal parameterization., IEEE Trans. Vis. Comput. Gr. 2006.

[15] Desbrun M, Kanso E, Tong Y, Discrete differential forms for computational modeling, Oberwolfach Seminars, Discrete Differential Geometry 2008.

[16] M. Mrozek, P. Pilarczyk, N. Żelazna, Homology Algorithm Based on Acyclic Subspace, Computers and Mathematics. 2008.

[17] M. Mrozek, B. Batko, Coreduction Homology Algorithm, Discrete and Computational Geometry, 2009.

[18] S. Day, W. D. Kalies, T. Wanner. Verified homology computations for nodal domains., SIAM Journal on Multiscale Modeling \& Simulation 2009.

[19] P. Dtotko, R. Specogna, Efficient cohomology computation for electromagnetic modeling, CMES: Computer Modeling in Engineering \& Sciences 2010.

[20] Rocio Gonzalez-Diaz, Adrian Ion Mabel, Iglesias-Ham, and Walter G. Kropatsch. Irregular graph pyramids and representative cocycles of cohomology generators. Lecture Notes In Computer Science archive Proceedings of the 7th IAPR-TC-15 International Workshop on Graph-Based Representations in Pattern Recognition, 2009.

[21] Thomas Lewiner, Helio Lopes, and Geovan Tavares. Optimal discrete morse functions for 2-manifolds. Computational Geometry: Theory and Applications, 2003. 